\title{
Ambulatory neurosurgery in the COVID-19 era: patient and provider satisfaction with telemedicine
}

\author{
Alina Mohanty, BA, ${ }^{1}$ Visish M. Srinivasan, MD, ${ }^{1}$ Jan-Karl Burkhardt, MD, ${ }^{1}$ Jeremiah Johnson, MD, ${ }^{1}$ \\ Akash J. Patel, MD, ${ }^{1}$ Sameer A. Sheth, MD, PhD, ${ }^{1}$ Ashwin Viswanathan, MD, ${ }^{1}$ Daniel Yoshor, MD, ${ }^{1}$ \\ and Peter Kan, MD²
}

\begin{abstract}
'Department of Neurosurgery, Baylor College of Medicine, Houston; and 2Department of Neurosurgery, University of Texas Medical Branch, Galveston, Texas
\end{abstract}

\begin{abstract}
OBJECTIVE Telemedicine has rapidly expanded in the recent years as technologies have afforded healthcare practitioners the ability to diagnose and treat patients remotely. Due to the COVID-19 pandemic, nonessential clinical visits were greatly limited, and much of the outpatient neurosurgical practice at the authors' institution was shifted quickly to telehealth. Although there are prior data suggesting that the use of telemedicine is satisfactory in other surgical fields, data in neurosurgery are limited. This study aimed to investigate both patient and provider satisfaction with telemedicine and its strengths and limitations in outpatient neurosurgery visits.

METHODS This quality improvement study was designed to analyze provider and patient satisfaction with telemedicine consultations in an outpatient neurosurgery clinic setting at a tertiary care, large-volume, academic center. The authors designed an 11-question survey for neurosurgical providers and a 13-question survey for patients using both closed 5-point Likert scale responses and multiple choice responses. The questionnaires were administered to patients and providers during the period when the clinic restricted in-person visits. At the conclusion of the study, the overall data were analyzed qualitatively and quantitatively.
\end{abstract}

RESULTS During the study period, 607 surveys were sent out to patients seen by telehealth at the authors' academic center, and 122 responses were received. For the provider survey, 85 surveys were sent out to providers at the authors' center and other academic centers, and 40 surveys were received. Ninety-two percent of patients agreed or strongly agreed that they were satisfied with that particular telehealth visit. Eighty-eight percent of patients agreed that their telehealth visit was more convenient for them than an in-person visit, but only $36 \%$ of patients stated they would like their future visits to be telehealth. Sixty-three percent of providers agreed that telehealth visits were more convenient for them than in-person visits, and $85 \%$ of responding providers stated that they wished to incorporate telehealth into their future practice.

CONCLUSIONS Although the authors' transition to telehealth was both rapid and unexpected, most providers and patients reported positive experiences with their telemedicine visits and found telemedicine to be an effective form of ambulatory neurosurgical care. Not all patients preferred telemedicine visits over in-person visits, but the high satisfaction with telemedicine by both providers and patients is promising to the future expansion of telehealth in ambulatory neurosurgery.

https://thejns.org/doi/abs/10.3171/2020.9.FOCUS20596

KEYWORDS ambulatory; COVID-19; telemedicine; provider; patient; satisfaction; survey

$\mathrm{T}$ ELEMEDICINE, the practice of using audio or visual communication technology to deliver care to a patient distantly, has expanded as technologies have afforded healthcare practitioners the ability to diagnose and treat patients remotely. Telehealth was first used in the early 1900 s to deliver medical advice over the radio. ${ }^{1}$ Since then, telehealth has become more commonplace, with one study in the Journal of the American Medical Association estimating an annual growth of 52\% between 2005 and
2015. ${ }^{2}$ However, telemedicine is more established in some medical specialties, such as psychiatry and primary care, than others. ${ }^{2}$ A study by the physician networking service Doximity listed neurosurgery as one of the 15 specialties least engaged in telemedicine job postings. ${ }^{3}$

Unprecedently, in December 2019, an outbreak of the SARS-CoV-2 virus caused a pandemic that was declared a public health emergency of international concern. ${ }^{4,5}$ The resulting COVID-19 was found to cause significant mortal- 
TABLE 1. Provider survey

\begin{tabular}{|c|c|c|c|c|c|}
\hline Survey Item & Strongly Disagree & Disagree & Neutral & Agree & Strongly Agree \\
\hline Telehealth is more convenient than in-person clinic. & 5 & 20 & 12.5 & 30 & 32.5 \\
\hline I prefer telehealth visits to in person visits. & 12.5 & 22.5 & 22.5 & 25 & 17.5 \\
\hline I am able to address patients' clinical needs adequately using telehealth. & 2.5 & 12.5 & 22.5 & 40 & 22.5 \\
\hline $\begin{array}{l}\text { I am able to do the relevant neurological exam virtually/My clinical exam is } \\
\text { comprehensive for the purposes of this visit. }\end{array}$ & 17.5 & 35 & 20 & 20 & 7.5 \\
\hline Communication with my patients is adequate when using telehealth. & 2.5 & 2.5 & 12.5 & 47.5 & 35 \\
\hline Enough time is spent with my patients when I use telehealth. & 2.5 & 0 & 12.5 & 45 & 40 \\
\hline Telehealth should be adapted more into my practice. & 5 & 5 & 7.5 & 37.5 & 45 \\
\hline I would like to do more telehealth visits in the future. & 5 & 2.5 & 7.5 & 37.5 & 47.5 \\
\hline Telehealth is a safe and effective method of seeing patients. & 2.5 & 5 & 10 & 37.5 & 45 \\
\hline
\end{tabular}

Values are percentages.

ity in those older than 60 years and in those with common underlying chronic conditions. ${ }^{4}$ The highly transmissible nature of the virus and the severity of the COVID-19 illness resulted in many states implementing a "Stay Home, Work Safe" policy in March 2020 to prevent the spread of the virus. ${ }^{6}$ As a result, nonessential clinical visits were limited, and much of our center's outpatient neurosurgical practice quickly shifted to telehealth. ${ }^{6}$

Although prior data suggest that the use of telemedicine is satisfactory in other surgical fields, the application of telemedicine in neurosurgery is in its infancy, and limited data exist measuring patient and provider satisfaction of telemedicine as well as its effectiveness in this field.? Therefore, this study aims to investigate patient and provider satisfaction with telemedicine in outpatient neurosurgery visits and to identify strengths and weaknesses of telemedicine as it pertains to ambulatory neurosurgery care. We hope to use this knowledge to optimize the use of telemedicine in neurosurgery ambulatory practice in the future.

\section{Methods}

This quality improvement study was designed to analyze the quality of telemedicine consultations in an outpatient neurosurgery clinic setting at a tertiary care, largevolume, academic center. Study data were collected and managed using REDCap electronic data capture tools. ${ }^{8,9}$ We designed an 11-question survey for neurosurgical providers primarily using questions with closed responses on a 5-point Likert scale (strongly disagree, disagree, neutral, agree, and strongly agree) (Table 1 and Fig. 1). The provider survey focused on the following areas: convenience (1 question), adequate time and communication (2 questions), current or future preferences for telehealth visits (3 questions), and effectiveness of the visit (i.e., adequately addressing patient's clinical needs or performing clinical examinations; 3 questions). Two further multiple-choice questions were included to identify the subspecialty of the neurosurgeon and what type of visit the provider believed most useful for telemedicine as opposed to an in-person visit.

For patients, we designed a 13-question survey using both closed 5-point Likert scale responses and multiple- choice responses (Tables 2 and 3 and Fig. 2). The survey addressed the following: convenience and satisfaction of the visit (3 questions), current and future preferences for telehealth ( 2 questions), the satisfaction of the provider's explanation of radiological findings (2 questions), the satisfaction with the clinical examination (2 questions), the ease at which telehealth technology was accessed (1 question), if adequate time was spent with the provider (1 question), what type of visit occurred (1 question), and if an upcoming surgery was discussed (1 question). Both providers and patients were also able to add comments about the visit with an open-ended response at the end of their surveys.

Both surveys were evaluated for face validity by 3 additional neurosurgical providers. The inclusion criterion for patient participants was that the patient was seen through a telehealth visit by a neurosurgical provider at our institution between March 1, 2020 (when telehealth first began in our department), and July 2, 2020 (before our department transitioned back to allowing all in-person visits). A telehealth visit for the purposes of this study is defined as either a scheduled video call or a telephone interaction that took the place of an in-person visit. The inclusion criterion for providers was that they were practicing telemedicine during the dates of our study (from March 1, 2020, to July 2,2020 ) at either our center or a similar academic tertiary care center in the country. Since surveys to patients were sent by the Epic Systems Corp. patient portal MyChart, the only exclusion criterion for the study was if patients did not have active MyChart accounts through which they could receive the survey.

The surveys for providers were sent out at one time to all practicing neurosurgeons in our academic center through an email link. Surveys to providers outside our academic center were sent out similarly. The patient surveys were first sent in one batch at the beginning of the study to all patients who were seen by telehealth at our single institution after March 1, 2020, but before the initiation of our study in early May, and then were sent out at the end of each week to the patients seen by telemedicine that week at the same institution. At the conclusion of the study, we analyzed the overall data. 


\section{What type of visit is telehealth the most useful for when compared to an in- person visit?}

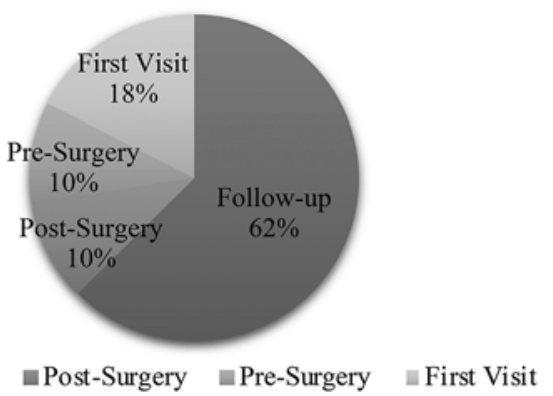

FIG. 1. Provider preference for type of visit performed by telehealth.

\section{Results}

With the patient survey, 607 surveys were sent, and 122 responses were received (response rate 20.1\%); 43 patients were excluded from the study because of an inactive MyChart account. For the provider survey, 85 surveys were sent out, and 40 surveys were received (response rate $47.1 \%$ ). In the 2 months prior to the shift to telemedicine, 26 patients per day were seen on average in clinic. The average daily clinical volume decreased approximately $50 \%$ from this baseline between March 22 and May 8, with 17 being the average daily clinical volume of patients while our study was taking place (Fig. 3).

Of our 40 providers, 16 respondents were vascular neurosurgeons, 6 neurosurgeons specialized in spine, 2 were functional neurosurgeons, 4 respondents identified as pediatric neurosurgeons, 3 were neurosurgical oncologists, 8 neurosurgeons selected more than one subspecialty, and 1 was a nurse practitioner. While $62 \%$ of providers agreed that a telehealth visit was more convenient for them than an inperson visit, only $42 \%$ agreed that they preferred telehealth to in-person visits. Sixty-two percent of providers agreed that they were able to adequately address patient's needs using telehealth, but $52 \%$ of providers disagreed that they were able to do the relevant neurological examination vir- tually. Eighty-three percent of providers believed that telehealth was a safe and effective method of seeing patients. Eighty-two percent of providers felt their communication with their patients was adequate when using telehealth, and $85 \%$ of providers agreed that they spent enough time with their patient. Eighty-two percent of providers stated that they would like to do more telehealth visits in the future, with $85 \%$ responding that they wished to incorporate telehealth in their future practice. When asked what type of visit telehealth was most suited for, $62 \%$ of providers chose follow-up visits, $18 \%$ chose first visits, $10 \%$ chose presurgery visits, and $10 \%$ chose postsurgery visits. To further analyze satisfaction and preferences for telehealth by subspecialty, the data were grouped by subspecialty (Table 4).

Follow-up visits were reported to be $38 \%$ of patient telehealth visits, with the next most common visit being first visits (26\%). Ninety-two percent of patients agreed that they were satisfied with their telehealth visit. Sixtynine percent of patients agreed that they were able to join the telehealth video call easily. Ninety percent of patients agreed that their provider spent enough time with them during the telehealth visit, and $91 \%$ of patients agreed that their provider satisfactorily addressed their clinical needs. Sixty-four percent of patients agreed that they were able to adequately show their clinical signs to their provider, while $72 \%$ agreed that their provider was able to sufficiently explain radiological findings to them over telehealth. Eighty-eight percent of patients agreed that their telehealth visit was more convenient for them than an in-person visit. Only $46 \%$ of patients agreed that they preferred telehealth visits to in-person visits, with another $40 \%$ stating they were neutral on the matter. Similarly, only $36 \%$ of patients stated they would like their future visits to be telehealth visits, with $48 \%$ patients stating they felt neutrally about this statement.

\section{Discussion}

Telemedicine has been well utilized in neurology, notably for the purposes of stroke evaluation and resultant alteplase (tissue plasminogen activator [tPA]) administration within a 4.5-hour window. Studies have shown that CT scans could be reliably interpreted, the National Institutes of Health Stroke Scale be accurately determined, and tPA

TABLE 2. Patient survey

\begin{tabular}{|c|c|c|c|c|c|}
\hline Survey Item & Strongly Disagree & Disagree & Neutral & Agree & Strongly Agree \\
\hline I was satisfied with my telehealth visit today. & 0 & 0.8 & 7.4 & 26.2 & 65.6 \\
\hline I was able to access the video link and join the telehealth video call easily. & 3.3 & 9.8 & 18 & 23 & 45.9 \\
\hline My provider spent enough time with me in my telehealth visit today. & 0 & 0 & 9.8 & 23.8 & 66.4 \\
\hline My provider was able to address all my clinical needs to my satisfaction. & 0.8 & 4.1 & 4.1 & 27.9 & 63.1 \\
\hline $\begin{array}{l}\text { I was able to show my provider my clinical signs through Zoom to my } \\
\text { satisfaction without seeing the provider in person (if applicable). }\end{array}$ & 4.2 & 7.6 & 24.4 & 19.3 & 44.5 \\
\hline $\begin{array}{l}\text { My provider explained my radiological findings through Zoom to my } \\
\text { satisfaction (if applicable). }\end{array}$ & 0.8 & 4.2 & 22.7 & 18.5 & 53.8 \\
\hline I prefer telehealth visits over in-person visits. & 4.1 & 9.1 & 40.5 & 24.8 & 21.5 \\
\hline I would like my future visits to be telehealth. & 3.4 & 12.7 & 48.3 & 16.1 & 19.5 \\
\hline
\end{tabular}

Values are percentages. 


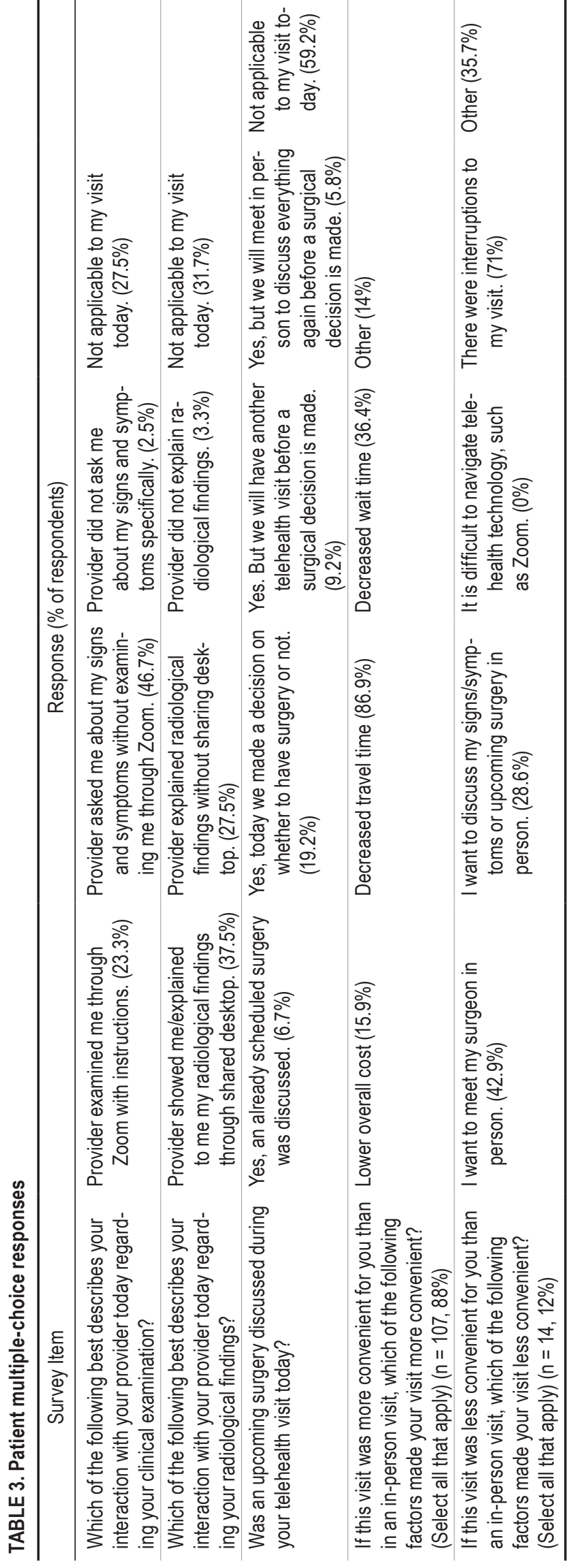

\section{Type of Visit}

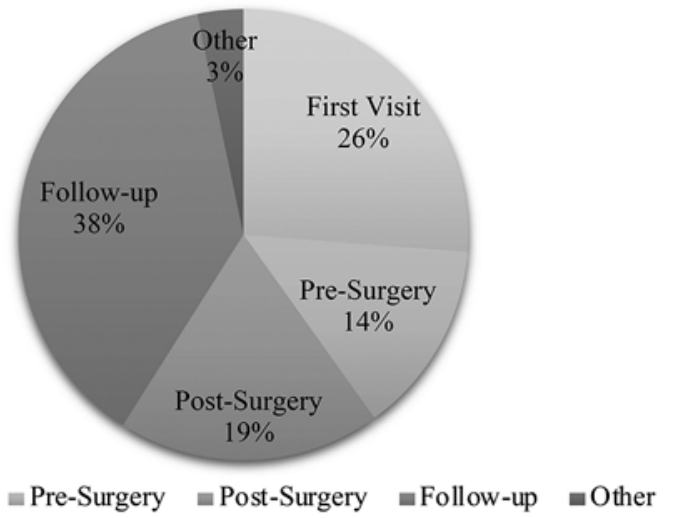

FIG. 2. Patient preference for type of visit performed by telehealth.

be safely administered to the patient through telehealth. ${ }^{10}$ Telestroke is especially beneficial in areas in which doorto-needle time is increased, e.g., in the case of a rural setting where there is low access to a stroke center. A trial from France implementing telestroke care in 10 regional emergency departments found that patients in their telehealth arm increased their tPA eligibility fivefold. ${ }^{11}$ Additionally, by receiving stroke care through telemedicine at a regional center, there is a significant cost reduction, estimated to be $\$ 4997$ per patient in one study, associated with decreased transfers to tertiary care centers. ${ }^{12}$ Aside from telestroke, telemedicine has been implemented in other fields of neurology such as tele-epilepsy and Parkinson's disease, where much of the diagnosis and monitoring of patients is dependent on history and can be elicited from a conversation through telehealth..$^{10,13}$

There exists sparse literature showing the application of telemedicine in neurosurgery; most of the literature that exists studied the used of telemedicine for follow-up visits. A 1200-patient study in rural India for postneurosurgical follow-up care found that telemedicine had higher perceived utility and was more cost-effective than routine care for the population studied..$^{14}$ In another study, telemedicine was implemented to monitor neurosurgical wounds using cell phone images.$^{15} \mathrm{~A}$ single-center prospective study investigating telemedicine for elective neurosurgical procedure follow-ups showed that the 47 patients in the telemedicine arm of the study had no change in the number of emergency department visits or readmission rates at 30 and 90 days postoperation compared with those who received in-person follow-up. ${ }^{16}$ Studies from countries such as Brazil and Albania have shown that telemedicine is an effective way to determine the severity of cases and the need for transfer in neurotrauma ${ }^{17,18}$ By triaging patients through a telehealth platform, access to specialty care in a remote or rural setting is increased, and costs are decreased by preventing unnecessary transfers that can be managed by nonneurosurgeons. ${ }^{17,18}$

Although telehealth has recently become more pervasive, it was only after COVID-19 that our department transitioned from primarily in-person clinic visits to telehealth for a wide variety of outpatient visits. Most importantly, 


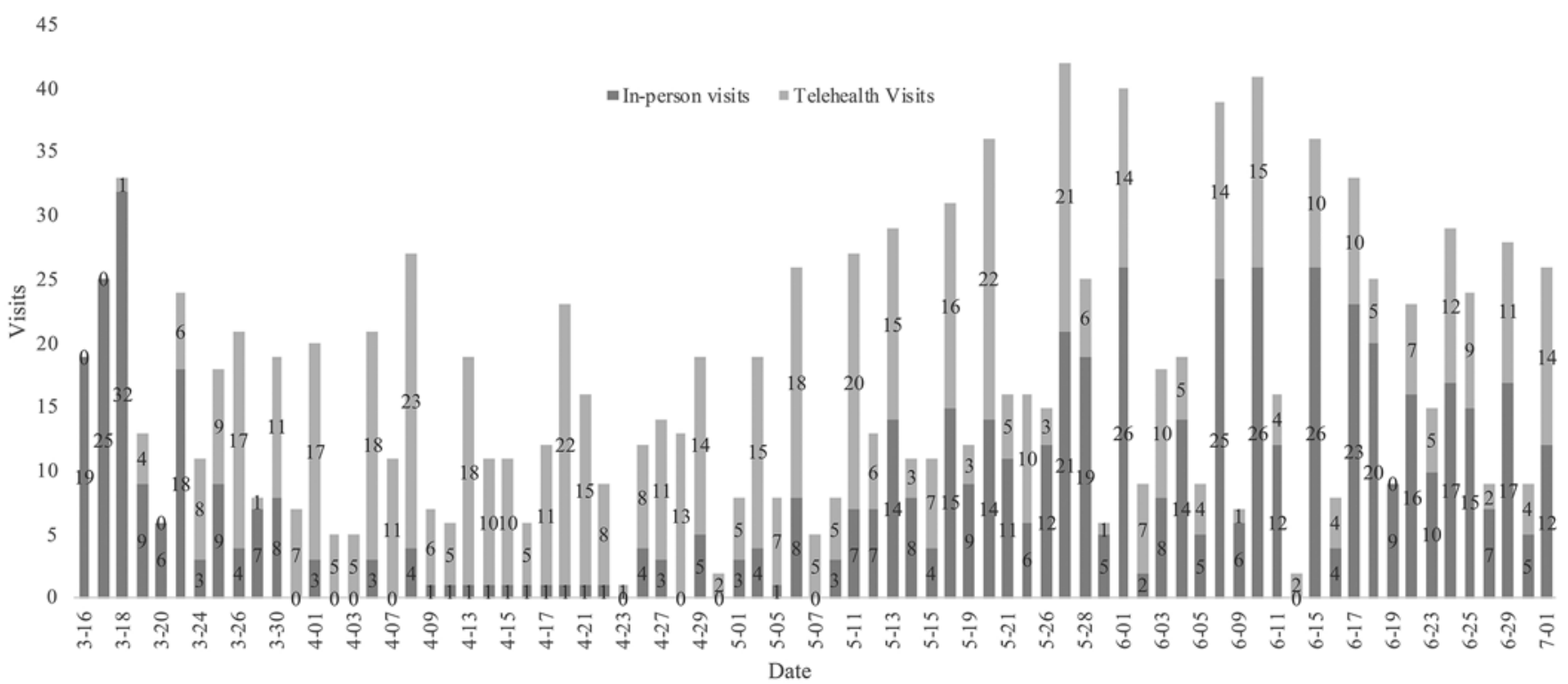

FIG. 3. Our center's clinical volume during the extent of the study.

by cancelling nonessential in-person hospital encounters, our patients had less contact with others in the hospital from whom they might be exposed to COVID-19. Second, by reducing the patient traffic through the hospital, we could redirect both human and material resources such as personal protective equipment toward efforts to combat COVID-19 and allow nonessential employees to stay home to reduce exposure to the virus.

Our center first conducted telehealth visits on March 18, 2020. Between March 30 and May 22, 2020, the majority of our patients were seen through telehealth. Although most of the telehealth visits were conducted by video, some were conducted through telephone. The choice of telephone or video depended on the both the patient and provider; in some cases, patients did not have access to video calling, and in other cases, providers felt that a telephone visit was satisfactory for the specific clinical encounter. Although clinical visits continued through both telehealth and face-to-face encounters, our clinical volume decreased significantly $(50 \%)$ during the stay-athome order. This decrease could largely be due to those who chose to reschedule or cancel appointments rather than meet their providers through telehealth.

In addition to the benefits of avoiding the hospital and thereby decreasing exposure to COVID-19, patients cited decreased travel and wait times as reasons why their visits were more convenient. In a study looking at telemedicine in pediatric postoperative urological care, the authors found that for every additional 23 miles of distance to the clinic, patients were $111 \%$ more likely to prefer remote follow-up..$^{19,20}$ Socioeconomic factors also played a role in preferring telehealth for some; one patient stated that they preferred telemedicine because they "[had] no ride," while another stated they "appreciated the ability to keep working." Many patients and their families have to travel to receive specialty care; a study from Florida for pediatric neurosurgery patients from southeast Georgia found that an average of $\$ 223$ per family was saved in work and transportation costs as a result of a telemedicine clinic. ${ }^{21}$

While $65 \%$ of providers were able to explain radiological findings with or without a shared desktop, one area in which providers found telehealth interactions inadequate was the physical examination. Neurosurgery remains one of the few fields in which the physical examination remains ever important, even in this day and age of imaging. In our survey, $72 \%$ of providers either disagreed or were neutral when asked if they were able to do the relevant clinical examination virtually or in a comprehensive manner. This could play a role in why most providers believed that follow-up visits were most suited for telehealth.

Although there exist overall trends in provider opinions in our survey, when splitting responses by subspecialty, we start to see some trends based on the subspecialty (Table 4). As discussed above, most providers found performing the clinical examination to be one of the more negative aspects of telemedicine. Some specialties, such as spine, rely on the examination much more than other specialties such as vascular, which can have asymptomatic patients monitored purely by imaging studies. Interestingly, despite spine surgeons being dissatisfied with the ability to perform a physical examination by telehealth, most of the spine surgeons surveyed agreed that they would like to incorporate telemedicine more into their future practice, with this group citing follow-ups as the most suitable appointment type for telemedicine. Although the clinical examination can be difficult to perform virtually, many methods exist to test components of the neurological examination. Blue et al. highlighted alternative methods to test these components, many of which include family members or tele-examiners for assistance. ${ }^{22}$ While patient cooperation can greatly aid the clinician in performing the neurological examination virtually, patient participation could be difficult in a subspecialty like pediatric neurosurgery, potentially explaining why all four of the pediatric 


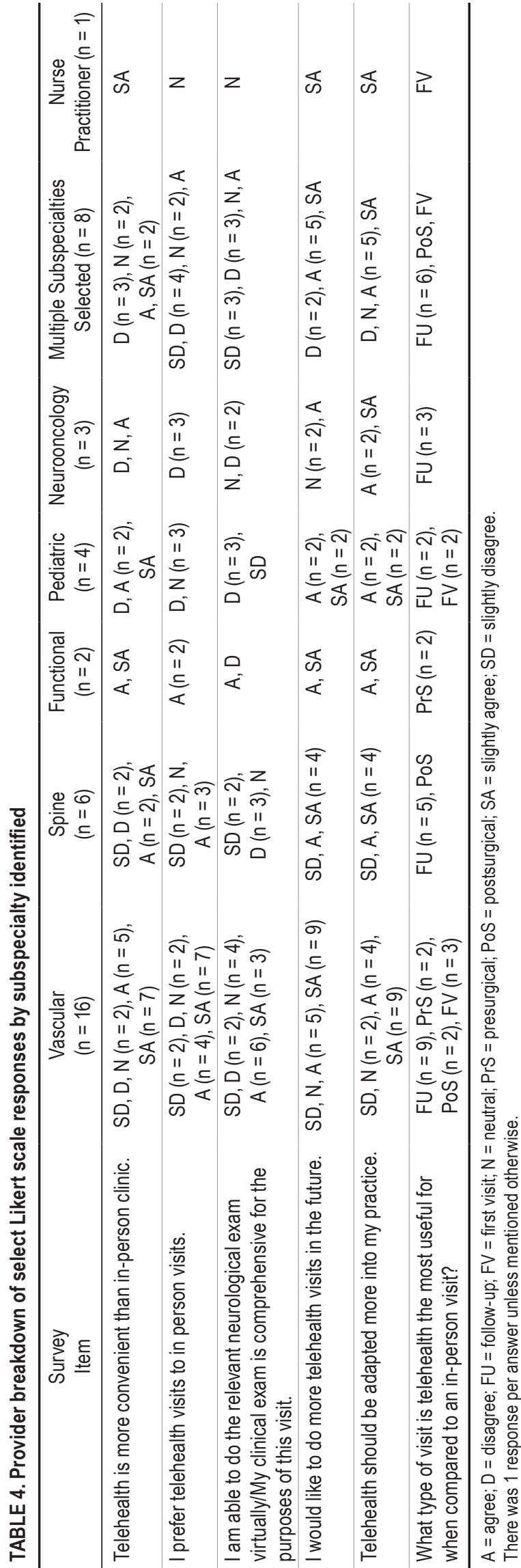

neurosurgeons surveyed were dissatisfied with their ability to perform their neurological examinations. Using these alternative and somewhat creative methods of performing the examination may not be able to replace an in-person clinical examination, but it might serve as a substitute should it need to be performed by telemedicine (Table 5).

Another factor in most providers preferring telemedicine for follow-up visits as opposed to first-time visits is to allow the development of the patient-doctor relationship. This relationship is vital in the field of neurosurgery, where a patient must have an inordinate trust to allow their doctor to perform potentially life-threatening surgery. Inperson encounters can provide more "assurance," in the words of one patient. Another patient stated, "[I] would support [telemedicine] for follow-up, but might be hesitant to. . for a new condition." One subspecialty in which this patient-surgeon relationship is incredibly important is neurosurgical oncology; perhaps for this reason, all three of the neurosurgical oncologists surveyed preferred inperson visits to telehealth visits. One subspecialty group that did not prefer follow-up appointments for telehealth was functional neurosurgery; both of our functional neurosurgeons stated that telehealth was most useful for presurgery visits. This could be explained by the fact that for functional neurosurgery, surgical success is often assessed in follow-up appointments by detailed examination.

Interestingly, from our survey, it seems that physicians are interested in incorporating telehealth into a larger part of their practice, but the same is not true for patients. Despite these factors listed above, providers overall agreed that telemedicine was a safe and effective way of seeing patients and that they would like to incorporate telemedicine more into their practice. Patients, on the other hand, remained largely neutral on whether they preferred future visits to be conducted via telemedicine, suggesting that, although telemedicine was more convenient, some other factors such as wanting to meet their doctor in person played a role in their not overtly preferring telemedicine to in-person visits.

There is no doubt that telemedicine will expand in the years to come. However, there remain challenges before it is adapted widely. Although the majority of our patients had no difficulties joining the video link for telehealth, using telemedicine might be challenging for those not wellacquainted with, or without possession of, the required technology, such as the elderly. A recent study in the Kaiser Permanente system showed that primary care patients older than 65 years were less likely to choose telemedicine than patients aged 18-44 years, suggesting that telemedicine might have a greater place in medical practice in the future with younger generations. ${ }^{23}$ As telemedicine becomes more widely practiced, it is possible that patients will seek out more second opinions, either on a local or national scale. Practitioners and health systems will have to invest in the infrastructure necessary to both market and facilitate telemedicine as a component of practice, including the training and maintenance of key personnel, staff, and technological support in addition to the actual technology. ${ }^{24}$ For telemedicine to be widely implemented, it must be cost-effective for all players involved in healthcare, be it the providers, insurers, or the patients themselves. 
Other barriers to incorporating telemedicine into neurosurgical practice include reimbursement policies and state-licensure laws. In the pre-COVID-19 era, the Centers for Medicare \& Medicaid Services (CMS) only offered Medicare reimbursement for telemedicine if the patient or beneficiary resided in a rural area and traveled to a local medical facility for the consultation. ${ }^{22,25}$ In response to COVID-19, the CMS, through the Coronavirus Preparedness and Response Supplemental Appropriations Act, 2000, and Section 1135 waiver, removed the requirement of receiving care at a designated health center. ${ }^{6,22}$ Additionally, the federal health programs Medicare, Medicaid, and Children's Health Insurance Plan reimbursed telemedicine visits at the same rate as in-person visits during the pandemic. 22,25 Despite these improvements to reimbursement policies, it remains to be seen whether these policies will remain in place after COVID-19. Another barrier is interstate license reciprocity; telemedicine can increase access to neurosurgical care in underserved rural areas, but practitioners not being licensed in the state where the patient is being seen poses an obstacle to telemedicine. Although the Interstate Medical Licensure Compact somewhat alleviates this issue by allowing qualified providers to have an expedited licensure process, this legislation has only been passed in 29 states. $^{22}$

Although many barriers to broad implementation of telemedicine in neurosurgical practice remain, telemedicine provides many benefits. One possibility of expanding telemedicine in neurosurgical practice is to allow both patients and providers the choice between in-person and telemedicine visits. If it is a first visit or a visit during which in-person discussion or a clinical examination is warranted, providers should be able to request an in-person visit. Perhaps for routine visits such yearly follow-ups in which a patient-doctor relationship has already been established, if both the practitioner and patient prefer, telemedicine could pose a convenient and efficient alternative to an in-person clinical encounter.

\section{Limitations}

Innate variability in both providers and patients can affect how satisfied a patient or provider is with a clinical interaction. Providers surveyed were from our academic institution as well as other academic institutions that had switched to telehealth. Because experiences vary across institutions, providers might have had different experiences with telehealth that influenced their opinions as reported in the survey. None of the neurosurgeons surveyed are employed in private practice, which comprises a large portion of neurosurgery. Similarly, although we surveyed many more patients than providers, our patient sample size is relatively modest and represents a restricted sample from one academic center.

One notable limitation is that all of our survey responses were sent postencounter and were voluntary. Therefore, self-selection bias is likely; patients who choose to participate in a telehealth visit are more likely to report a positive experience.

Another possible unique limitation is that COVID-19 might have influenced preferences toward telehealth visits for increased patient and provider safety. It is possible that
TABLE 5. Age distribution of all telehealth patients seen at our institution

\begin{tabular}{cc}
\hline Age Range, Yrs & \% Patients \\
\hline $18-27$ & 6.7 \\
\hline $28-37$ & 11.3 \\
\hline $38-47$ & 12.4 \\
\hline $48-57$ & 20.0 \\
\hline $58-67$ & 25.3 \\
\hline $68-77$ & 19.3 \\
\hline $78-87$ & 4.4 \\
\hline $88-97$ & 0.6 \\
\hline
\end{tabular}

once the pandemic passes, patients would prefer in-person visits to telemedicine visits. Despite all these possible limitations, we believe that our survey offers valuable data on the perceptions of both patients and providers toward telehealth in the field of neurosurgery.

\section{Conclusions}

Although our transition to telehealth was both rapid and unexpected, most providers and patients reported positive experiences with telemedicine visits and found telemedicine to be an effective way to deliver ambulatory neurosurgical care. In our situation, using telemedicine in our practice greatly helped protect both patients and staff and reduce the transmission of COVID-19. Further and larger studies are needed to assess the quality of telehealth clinical encounters to show that telehealth visits are noninferior to in-person visits and to see which patient populations are most willing to participate in telehealth. Although not all patients preferred telemedicine visits over in-person visits, the high satisfaction with telemedicine by both providers and patients is promising in regard to the future expansion of telehealth. Only time will tell to what extent telehealth will be adapted in neurosurgical practice.

\section{Acknowledgments}

We acknowledge the contributions of Eddie Dinh to the survey data collection.

\section{References}

1. Institute of Medicine. The Role of Telehealth in an Evolving Health Care Environment: Workshop Summary. National Academies Press; 2012.

2. Barnett ML, Ray KN, Souza J, Mehrotra A. Trends in telemedicine use in a large commercially insured population, 2005-2017. JAMA. 2018;320(20):2147-2149.

3. Doximity. 2019 Telemedicine and Locum Tenens Opportunities Study-Measuring Physician Interest in Emerging Employment Areas. Published July 2019. Accessed October 15, 2020. https://s3.amazonaws.com/s3.doximity.com/press/2019 TelemedicineAndLocumTenensOpportunitiesStudy.pdf

4. Chen WH, Strych U, Hotez PJ, Bottazzi ME. The SARSCoV-2 vaccine pipeline: an overview. Curr Trop Med Rep. Published online March 3, 2020. doi:10.1007/s40475-02000201-6 
5. The Lancet Infectious Diseases. COVID-19, a pandemic or not? Lancet Infect Dis. 2020;20(4):383.

6. LoPresti MA, McDeavitt JT, Wade K, et al. Telemedicine in neurosurgery - a timely review. Letter. Neurosurgery. 2020; 87(2):E208-E210.

7. Snyder SR. Telemedicine for elective neurosurgical routine follow-up care: a promising patient-centered and cost-effective alternative to in-person clinic visits. Editorial. Neurosurg Focus. 2018;44(5):E18.

8. Harris PA, Taylor R, Thielke R, et al. Research electronic data capture (REDCap) - a metadata-driven methodology and workflow process for providing translational research informatics support. J Biomed Inform. 2009;42(2):377-381.

9. Harris PA, Taylor R, Minor BL, et al. The REDCap consortium: building an international community of software platform partners. J Biomed Inform. 2019;95:103208.

10. Patel UK, Malik P, DeMasi M, et al. Multidisciplinary approach and outcomes of tele-neurology: a review. Cureus. 2019;11(4):e4410.

11. Mazighi M, Meseguer E, Labreuche J, et al. TRUST-tPA trial: telemedicine for remote collaboration with urgentists for stroke-tPA treatment. J Telemed Telecare. 2017;23(1): 174-180.

12. Al Kasab S, Almallouhi E, Debenham E, et al. Beyond acute stroke: rate of stroke transfers to a tertiary centre following the implementation of a dedicated inpatient teleneurology network. J Telemed Telecare. Published online August 28, 2019. doi:10.1177/1357633X19868097

13. Schneider RB, Biglan KM. The promise of telemedicine for chronic neurological disorders: the example of Parkinson's disease. Lancet Neurol. 2017;16(7):541-551.

14. Thakar S, Rajagopal N, Mani S, et al. Comparison of telemedicine with in-person care for follow-up after elective neurosurgery: results of a cost-effectiveness analysis of 1200 patients using patient-perceived utility scores. Neurosurg Focus. 2018;44(5):E17.

15. Pirris SM, Monaco EA III, Tyler-Kabara EC. Telemedicine through the use of digital cell phone technology in pediatric neurosurgery: a case series. Neurosurgery. 2010;66(5): 999-1004.

16. Reider-Demer M, Raja P, Martin N, et al. Prospective and retrospective study of videoconference telemedicine followup after elective neurosurgery: results of a pilot program. Neurosurg Rev. 2018;41(2):497-501.

17. Esteves LA, Ribeiro AT, da Silva EG, et al. Evaluation of safety, effectiveness and reproducibility of telemedicine for neurosurgical screening. Einstein (Sao Paulo). 2019;17(4): eAO4609.

18. Olldashi F, Latifi R, Parsikia A, et al. Telemedicine for neurotrauma prevents unnecessary transfers: an update from a nationwide program in Albania and Analysis of 590 patients. World Neurosurg. 2019;128:e340-e346.
19. Gunter RL, Chouinard S, Fernandes-Taylor S, et al. Current use of telemedicine for post-discharge surgical care: a systematic review. J Am Coll Surg. 2016;222(5):915-927.

20. Canon S, Shera A, Patel A, et al. A pilot study of telemedicine for post-operative urological care in children. $J$ Telemed Telecare. 2014;20(8):427-430.

21. Hayward K, Han SH, Simko A, et al. Socioeconomic patient benefits of a pediatric neurosurgery telemedicine clinic. $J$ Neurosurg Pediatr. 2020;25(2):204-208.

22. Blue R, Yang AI, Zhou C, et al. Telemedicine in the era of coronavirus disease 2019 (COVID-19): a neurosurgical perspective. World Neurosurg. 2020;139:549-557.

23. Reed ME, Huang J, Graetz I, et al. Patient characteristics associated with choosing a telemedicine visit vs office visit with the same primary care clinicians. JAMA Netw Open. 2020;3(6):e205873.

24. Kahn EN, La Marca F, Mazzola CA. Neurosurgery and telemedicine in the United States: assessment of the risks and opportunities. World Neurosurg. 2016;89:133-138.

25. Contreras CM, Metzger GA, Beane JD, et al. Telemedicine: patient-provider clinical engagement during the COVID-19 pandemic and beyond. J Gastrointest Surg. 2020;24(7): 1692-1697.

\section{Disclosures}

The authors report no conflict of interest concerning the materials or methods used in this study or the findings specified in this paper.

\section{Author Contributions}

Conception and design: Kan, Mohanty. Acquisition of data: Kan, Mohanty. Analysis and interpretation of data: Kan, Mohanty. Drafting the article: Kan, Mohanty. Critically revising the article: all authors. Reviewed submitted version of manuscript: all authors. Approved the final version of the manuscript on behalf of all authors: Kan.

\section{Correspondence}

Peter Kan: University of Texas Medical Branch, Galveston, TX. ptkan@utmb.edu. 